\title{
Homology modeling and identification of amino acids involved in the catalytic process of Mycobacterium tuberculosis serine acetyltransferase
}

\author{
JUANJUAN QIU ${ }^{1}$, SHIZHU ZANG ${ }^{2}$, YUFANG MA ${ }^{3}$, LAWRENCE OWUSU $^{2}$, LEI ZHOU ${ }^{4}$, TAO JIANG ${ }^{2}$ and YI XIN ${ }^{2}$ \\ ${ }^{1}$ Centralab, The First Affiliated Hospital of Dalian Medical University, Dalian, Liaoning 116011; \\ Departments of ${ }^{2}$ Biotechnology and ${ }^{3}$ Biochemistry and Molecular Biology, Dalian Medical University, Dalian, \\ Liaoning 116044; ${ }^{4}$ Clinical Laboratory, Dalian Blood Centre, Dalian, Liaoning 116001, P.R. China
}

Received December 4, 2015; Accepted November 29, 2016

DOI: $10.3892 / \mathrm{mmr} .2017 .6156$

\begin{abstract}
Serine acetyltransferase (CysE) belongs to the hexapeptide acetyltransferase family and is involved in the biosynthesis of L-cysteine in microorganisms. Mycobacterium tuberculosis CysE is regarded as a potential target for anti-tuberculosis (TB) drugs; however, the structure and active sites of $M$.tuberculosis CysE remain unknown. The present study aimed to predict the secondary structure and to construct a 3D model for M.tuberculosis CysE using bioinformatics analysis. To determine the essential amino acids that are associated with CysE enzymatic activity, amino acid sequences from several microorganisms were compared, and a consensus sequence was identified. Subsequently, site-directed mutagenesis was used to generate mutant M.tuberculosis $\mathrm{CysE}$ proteins. Enzyme assays demonstrated that D67A, H82A and H117A mutants abolished $\sim 75 \%$ activity of $M$. tuberculosis CysE. Prediction of the protein structure and identification of the active amino acids for M.tuberculosis CysE is essential for designing inhibitors, which may aid the discovery of effective anti-TB drugs.
\end{abstract}

\section{Introduction}

Tuberculosis (TB) is caused by Mycobacterium tuberculosis infection and remains one of the major infectious diseases affecting people worldwide, particularly in developing countries. The morbidity and mortality of TB has been exacerbated by the human immunodeficiency virus, the emergence of multidrug-resistant TB, and extensively drug-resistant strains

Correspondence to: Professor Yi Xin, Department of Biotechnology, Dalian Medical University, 9 West Lvshun South Road, Dalian, Liaoning 116044, P.R. China

E-mail: xinyi20150707@126.com; jimxin@hotmail.com

Key words: Mycobacterium tuberculosis, serine acetyltransferase, homology modeling, site-directed mutagenesis, active amino acid of M.tuberculosis (1). Therefore, it is important to understand the disease and to investigate novel anti-TB drugs.

Unlike the synthesis of cysteine from methionine through homocysteine in fungi and animals (2), serine acetyltransferase (CysE) catalyzes the conversion of acetyl-coenzyme A (CoA) and L-serine to $\mathrm{CoA}$ and $O$-acetyl-L-serine, which is the first product and a key amino acid in the biosynthesis of L-cysteine in microorganisms and plants $(3,4)$. Cysteine is required for the biosynthesis of essential compounds, including L-methionine, thiamine, biotin, coenzyme A and other sulfur-containing compounds. Notably, CysE has no known biological function in humans, as different pathways are used in the anabolism of cysteine in microorganisms and humans. The M. tuberculosis CysE is therefore considered as a potential anti-TB drug target $(5,6)$. Our previous study demonstrated that the Rv2335 protein was the M.tuberculosis homolog of CysE, encoded by the cysE gene, and determined and described the kinetic parameters and optimal catalytic conditions of the enzyme (7). However, to the best of our knowledge, there is currently no information regarding the structure and active sites of M. tuberculosis CysE. Determination of the protein structure is crucial for investigating the relationship between structure and function of any protein. Homology modeled structures may characterize conserved domains and active amino acids, which are essential to the function of the protein.

In the present study, the CysE amino acid sequence from five species, M. tuberculosis, Escherichia coli, Salmonella typhimurium, Haemophilus influenza and Entamoeba histolytica, was compared. This comparison allowed for the prediction of the secondary structure of M. tuberculosis CysE and the construction of a 3D model. Three residues, D67, H82 and H117, were identified as significant sites for activity. Such identifications and characterizations may facilitate screening for enzyme inhibitors to catalytic processes in M. tuberculosis, and thus therapy for patients with TB.

\section{Materials and methods}

Materials. Reagents and enzymes involved in gene cloning and site-directed mutagenesis were purchased from Takara Bio, 
Inc. (Otsu, Japan). The chemicals for all enzyme assays were purchased from Sigma-Aldrich (Merck Millipore, Darmstadt, Germany). The Ni-NTA agarose column used for protein purification was obtained from Qiagen $\mathrm{GmbH}$ (Hilden, Germany).

Sequence analysis and alignments. The amino acid sequences of $M$. tuberculosis CysE (NP_216851) were retrieved from the National Center for Biotechnology Information (NCBI) GenBank database (Bethesda, MD, USA) (8). The ProtParam tool in the ExPASy server (http://web.expasy. org/protparam) was used to predict the physiochemical properties of M. tuberculosis CysE (9). The parameters included: Molecular weight, theoretical isoelectric point (pI), amino acid composition, atomic composition, extinction coefficient, estimated half-life, instability index, aliphatic index and grand average of hydropathy (GRAVY). CysE protein sequences of M. tuberculosis CysE, E. coli CysE (AJF44936), S. typhimurium CysE (KPF36926.1), H. influenzae CysE (KMZ31837.1) and E. histolytica CysE (BAA82868.1) were aligned using the MultAlin sequence alignment tool (http://multalin.toulouse.inra.fr/multalin) (10).

Secondary structure prediction and functional annotation of the M.tuberculosis CysE protein. Based on sequence similarity searches with orthologous family members, several bioinformatics tools were used to analyze the conserved domains of CysE: The European Bioinformatics Institutes' (EBI) InterProScan tool, which combines different protein signature recognition methods from InterPro consortium member databases into one resource (11); the University College London's PSIPRED protein structure prediction server (http://bioinf. cs.ucl.ac.uk/psipred), which is based on a neural network algorithm for predicting structural information about a protein from its amino acid sequence alone (12); and the NCBI Conserved Domains Database (NCBI-CDD; https://www.ncbi.nlm.nih. gov/cdd), which is a protein annotation resource that consists of a collection of well-annotated multiple sequence alignment models for ancient domains and full-length proteins (13).

$3 D$ modeling of CysE protein. A 3D structure of the $M$. tuberculosis CysE protein was modeled by comparative protein modeling methods using the SWISS-MODEL server (http://swissmodel.expasy.org) (14). A suitable template for homology modeling was selected using NCBI Position Specific Iterated-Basic Local Alignment Search Tool (PSI-BLAST) (15) against the Research Collaboratory for Structural Bioinformatics Protein Data Bank (PDB) (http://rcsb.org). The ExPASy Swiss-PdbViewer 3.5 (http://spdbv.vital-it.ch) was used to produce a structure-based alignment and SWISS-MODEL was used in the optimized mode to minimize energy $(14,16)$. For structural evaluation and stereochemical analyses, the 3D model was submitted to the EBI PDBsum pictorial database (17). The M.tuberculosis CysE protein model was further evaluated using the University of California, Los Angeles-Department of Energy Institute programs PROCHECK (18), Verify3D and ERRAT (http://services.mbi.ucla.edu).

Site-directed mutagenesis of M. tuberculosis cysE. Three pairs of mutated primers were designed according to the DNA sequence of $M$.tuberculosis cysE (Table I). Every pair mutated primer was characterized with their 5'ends adjacent and 3 'ends in opposite directions according to the instructions of the Takara MutanBEST kit (Takara Bio, Inc.). The recombinant plasmid pMD18-Mtb-cysE, which contains NdeI and XhoI restriction sites, was constructed previously (7) and was used as the template. The linear mutated plasmids were amplified using $P f u$ DNA polymerase (Thermo Fisher Scientific, Waltham, MA, USA). The amplification reaction was performed in a final volume of $50 \mu \mathrm{l}$ mixture including $0.5 \mu \mathrm{l} P f u$ DNA polymerase (5 U/ $\mu \mathrm{l}), 5 \mu \mathrm{l} P f u$ Buffer (10X), $5 \mu \mathrm{l}$ dNTP mixture (2.5 mM each), $1 \mu$ l template DNA (100 ng), $3 \mu$ l mutated primers $(20 \mu \mathrm{M})$ and $\mathrm{ddH}_{2} \mathrm{O}$. The reaction conditions consisted of pre-denaturation $\left(94^{\circ} \mathrm{C}, 5 \mathrm{~min}\right), 30$ cycles of amplification (denaturation at $94^{\circ} \mathrm{C}$ for $30 \mathrm{sec}$, annealing at $65 / 61^{\circ} \mathrm{C}$ for $30 \mathrm{sec}$ and primer extension at $72^{\circ} \mathrm{C}$ for $5 \mathrm{~min}$ ) and final extension at $72^{\circ} \mathrm{C}$ for $10 \mathrm{~min}$. Plasmids were recircularized by blunting kination ligation and self-ligation using Takara MutanBEST kit (Takara Bio, Inc.). pMD18-Mtb-cysE plasmids containing site-directed mutations were transformed and grown in E. coli Novablue competent cells (Merck Millipore). The plasmid was transformed into competent cells by heat shock at $42^{\circ} \mathrm{C}$. The E. coli Novablue cells harboring plasmid were cultured in lysogeny medium supplemented with $100 \mathrm{ug} / \mathrm{ml}$ ampicillin (Merck Millipore) at $37^{\circ} \mathrm{C}$. Positive clones were identified by restriction endonuclease digestion (Takara Bio, Inc.) and DNA sequencing as those harboring the required mutations. The sequencing work was collaborated with Takara Bio Inc.

Preparation of mutant $M$. tuberculosis CysE proteins. Site-mutated $M$. tuberculosis cysE genes were subcloned into pET29b (Merck Millipore) at NdeI and XhoI restriction sites, and transformed into E. coli BL21 (DE3) cells (Merck Millipore) to express the three different CysE mutated proteins using the heat shock method, each containing a polyhistidine (His)-tag. CysE mutant proteins were prepared and purified using $\mathrm{Ni}^{2+}$ affinity chromatography according to the expression protocol of wild-type M. tuberculosis CysE protein, as previously described (7). Total protein ( 10 mg) was loaded onto a $1 \mathrm{ml}$ Ni-NTA agarose column (Qiagen, Hilden, Germany). Then the column was washed with $20 \mathrm{ml}$ wash buffer (20 mM Tris- $\mathrm{HCl} \mathrm{pH} 8.0,500 \mathrm{mM} \mathrm{NaCl}, 20 \%$ glycerol, $60 \mathrm{mM}$ imidazole and $1 \mathrm{mM}$ PMSF). Finally, the mutant CysE proteins with a His-tag at the C-terminus were eluted with $10 \mathrm{ml}$ elution buffer $(20 \mathrm{mM}$ Tris- $\mathrm{HCl} \mathrm{pH} 8.0$, $500 \mathrm{mM} \mathrm{NaCl}, 20 \%$ glycerol, $300 \mathrm{mM}$ imidazole and $1 \mathrm{mM}$ PMSF). The concentration of purified mutant CysE proteins was measured using the BCA Protein Assay Kit (P0012S; Beyotime Institute of Biotechnology, Haimen, China). The purified mutant CysE proteins were identified by western blot analysis. The protocol was as followed: Purified proteins $(20 \mu \mathrm{g})$ per lane were loaded and sepearated by $12 \%$ SDS-PAGE (P0012A; Beyotime Institute of Biotechnology). The proteins were transferred to nitrocellulose membranes (Thermo Fisher Scientific, Inc.) after electrophoresed with SDS-PAGE. Following blocking in 5\% skimmed milk buffer for $2 \mathrm{~h}$ at $37^{\circ} \mathrm{C}$, the membrane was incubated with mouse antipolyhistidine monoclonal antibody (dilution, 1:5,000; H1029; Merck Millipore) for $2 \mathrm{~h}$ at $37^{\circ} \mathrm{C}$ and followed by horse-anti-mouse $\mathrm{IgG}$ conjugated with alkaline phosphatase 
A
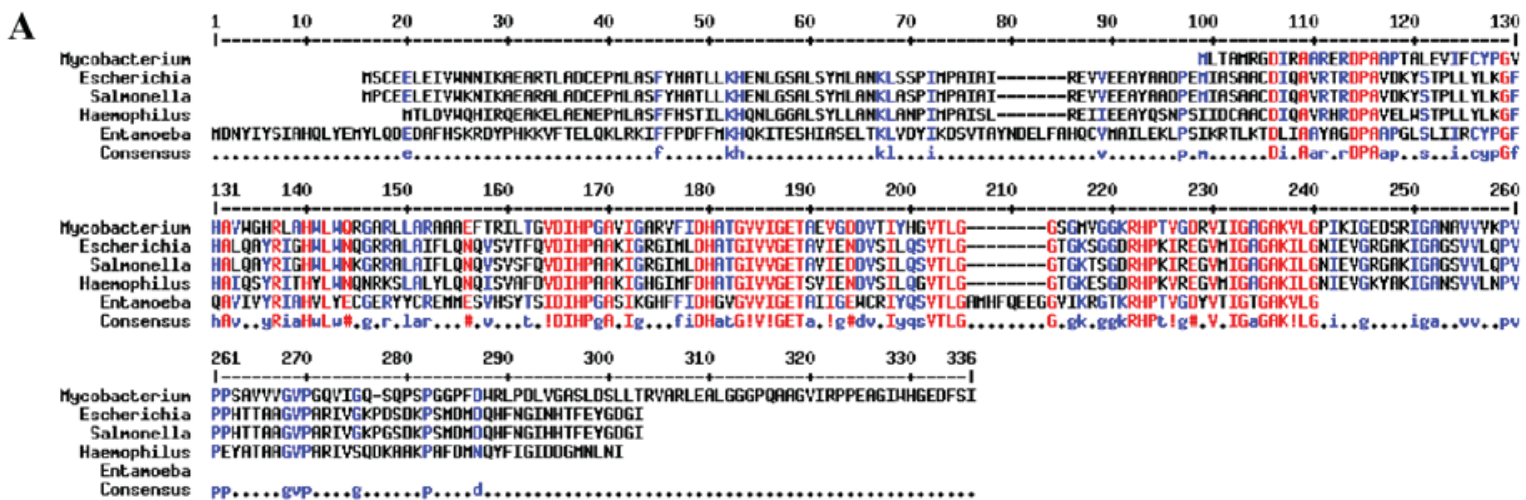

B

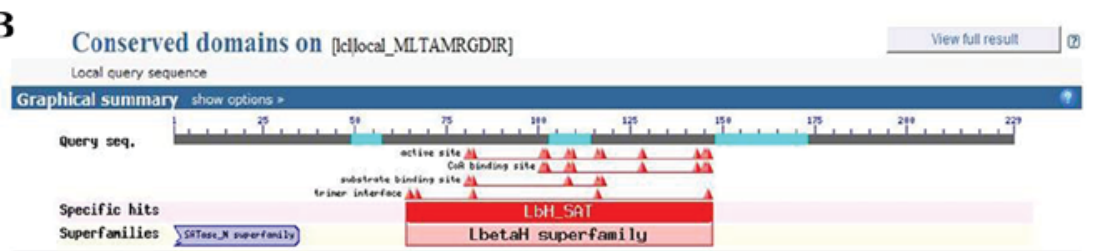

D
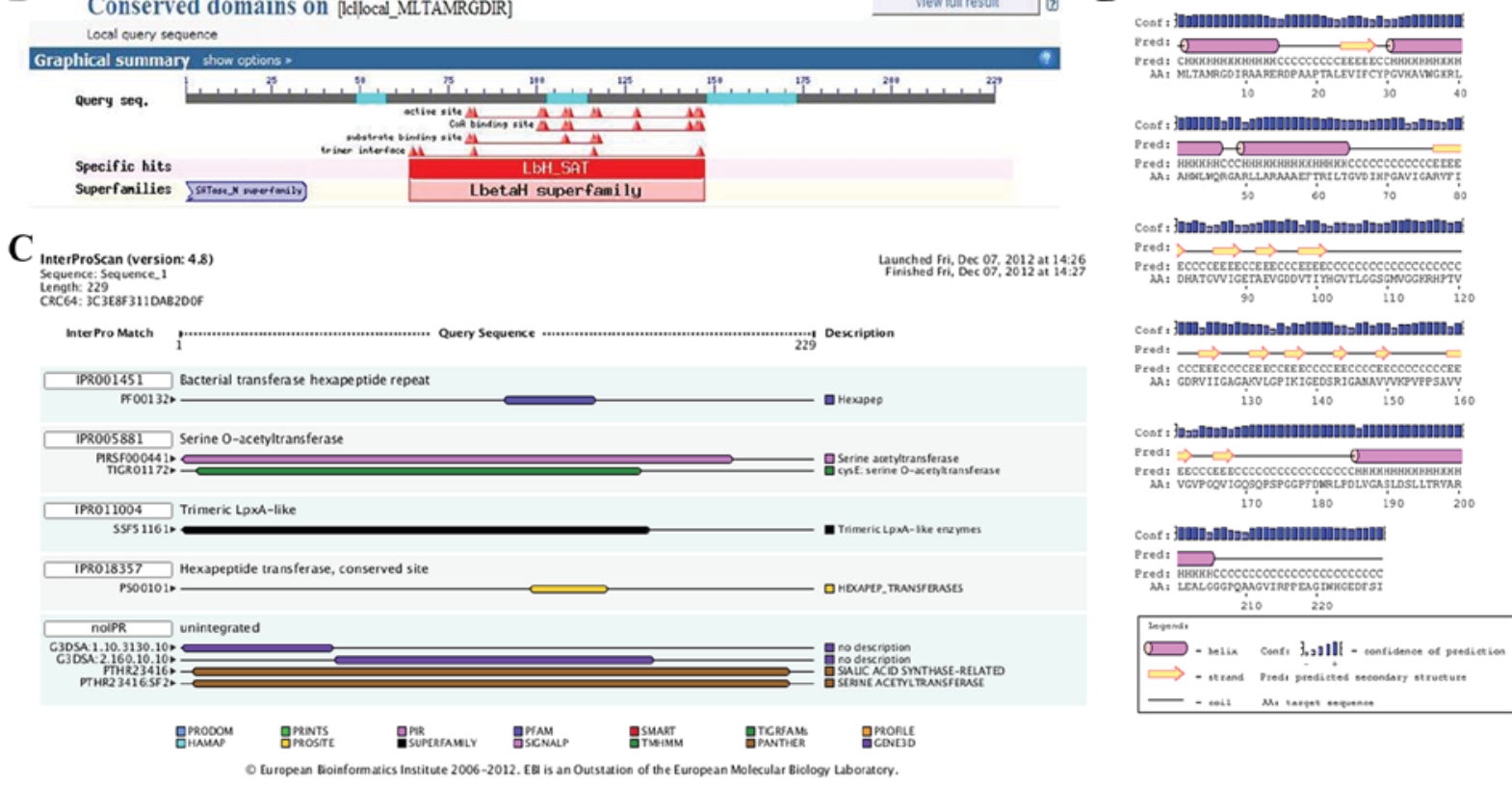

Figure 1. Amino acid sequence alignment and secondary structure prediction of M. tuberculosis CysE protein (A) Amino acid sequence alignment of homologous CysE proteins from Mycobacterium tuberculosis, Escherichia coli, Salmonella typhimurium, Haemophilus influenza and Entamoeba histolytica. Analysis of the conserved domains of CysE protein using (B) NCBI-CDD, (C) InterProScan and (D) PSIPRED. CysE, serine acetyltransferase; NCBI-CDD, National Center for Biotechnology Information-Conserved Domains Database.

(dilution, 1:2,000; ZB-2310; ZSGB-Bio, Beijing, China) for $45 \mathrm{~min}$ at room temperature. After staining in the solution mixture of BCIP/NBT (ZSGB-Bio), the immunoreactive protein bands were visualized and collected.

Enzymatic activity assay for mutant CysE proteins. The enzymatic activity assay of the various CysE mutants was conducted by the Ellman's reagent [5,5'-dithiobis-(2-nitrobenzoic acid); DTNB] method, as previously described (19). Briefly, equal concentrations of the purified CysE mutant proteins and the wild-type $M$. tuberculosis CysE protein were used. The relative activities of the mutated CysE proteins were calculated against activity of the wild-type M. tuberculosis CysE, which was used as the control (set to 100\%). All data were analyzed using GraphPad Prism 6 (GraphPad, La Jolla, CA, USA).

\section{Results and Discussion}

Sequence analysis and alignment. ProtParam was used to analyze various physiochemical properties from the amino acid sequence of CysE protein. The molecular weight and $\mathrm{pI}$ of the 229-amino-acid-long sequence were predicted as 23,770.3 Da and $\mathrm{pH} 7.11$, respectively. An instability index of 27.56 indicated that the CysE protein was stable. The GRAVY index of 0.217 was indicative of a hydrophobic protein. Sequence alignment demonstrated that the M.tuberculosis CysE protein shared identity with E. coli CysE (45\%), S. typhimurium CysE (45\%), H. influenzae CysE (45\%) and E. histolytica CysE (49\%) (Fig. 1A).

Secondary structure prediction and functional annotation of CysE protein. The bioinformatics tools InterProScan, PSIPRED and NCBI-CDD were used to search the conserved domains and potential functions of the M. tuberculosis CysE protein. The results revealed that the CysE protein possessed a left-handed- $\beta$-helix $(\mathrm{L} \beta \mathrm{H})$ domain and belonged to the hexapeptide acetyltransferase family (Fig. 1B-D), as previously shown for Rhizobium leguminosarm (20). This family of acetyltransferases is characterized by imperfect tandem repeats of the hexapeptide motif [LIV]-[GAED]-X2-[STAV]-X (21) 
Table I. Sequences of oligonucleotide primers used for constructing site-direct mutations of $M$. tuberculosis cysE gene.

\begin{tabular}{|c|c|c|}
\hline Name & $\begin{array}{l}\text { Primer } \\
\text { orientation }\end{array}$ & $\begin{array}{l}\text { Primer sequence }^{\mathrm{a}} \\
\qquad\left(5^{\prime}-3^{\prime}\right)\end{array}$ \\
\hline \multirow[t]{2}{*}{ D67A } & $\mathrm{F}$ & $\begin{array}{l}\text { ATCCTGACCGGTGTAGCCATCC } \\
\text { ACCCCGGTGCC }\end{array}$ \\
\hline & $\mathrm{R}$ & TACACCGGTCAGGATGCG \\
\hline \multirow[t]{2}{*}{$\mathrm{H} 82 \mathrm{~A}$} & $\mathrm{~F}$ & $\begin{array}{l}\text { CGCGTGTTCATCGACGCCGCG } \\
\text { ACCGGCGTGGTG }\end{array}$ \\
\hline & $\mathrm{R}$ & GTCGATGAACACGCGAGCACCG \\
\hline \multirow[t]{2}{*}{ H117A } & $\mathrm{F}$ & $\begin{array}{l}\text { GTTGGCGGGAAACGCGCCCCC } \\
\text { ACCGTCGGTGAC }\end{array}$ \\
\hline & $\mathrm{R}$ & GCGTTTCCCGCCAACCATGCC \\
\hline
\end{tabular}

${ }^{a}$ Mutated residues are underlined. F, forward; R, reverse.

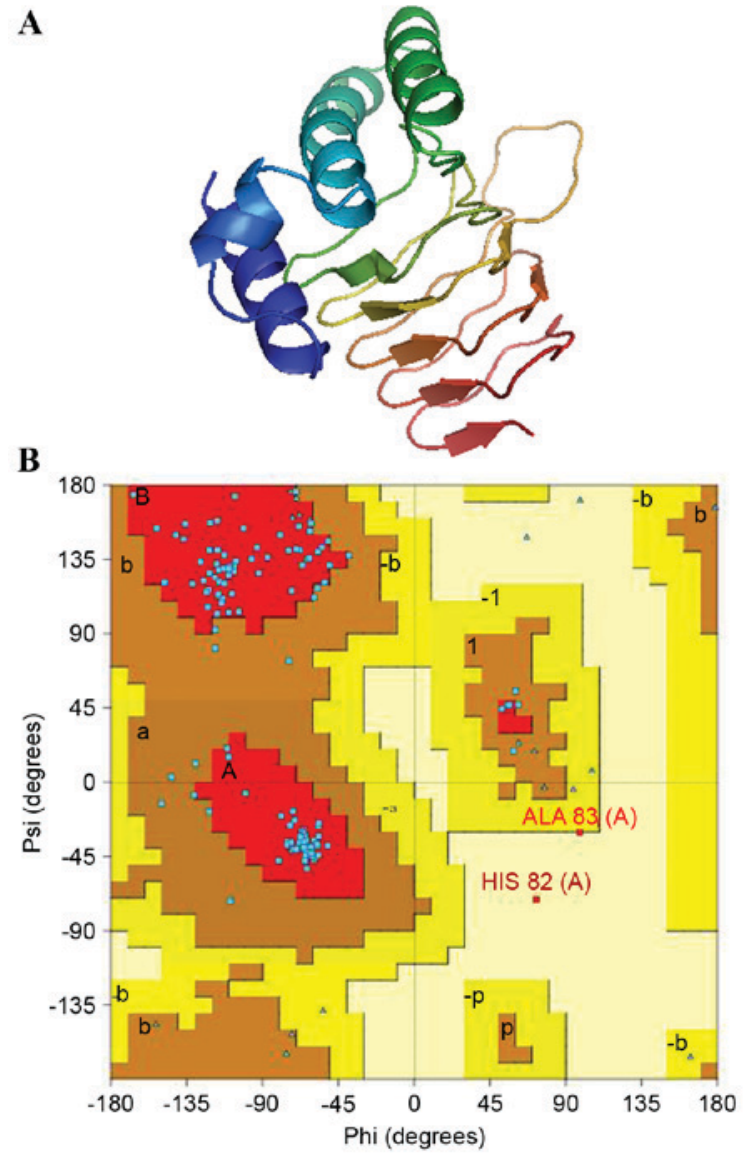

Figure 2. 3D structure prediction and assessment of M.tuberculosis CysE protein (A) Predicted 3D structure of CysE protein. (B) Ramachandran plot for the predicted model of CysE. CysE, serine acetyltransferase.

with a conserved $\mathrm{L} \beta \mathrm{H}$ domain, which is folded by three hexapeptides $(22,23)$.

$3 D$ modeling of the CysE protein. Homology modeling is a standard method for structure prediction and contributes to the understanding of the relationship between protein

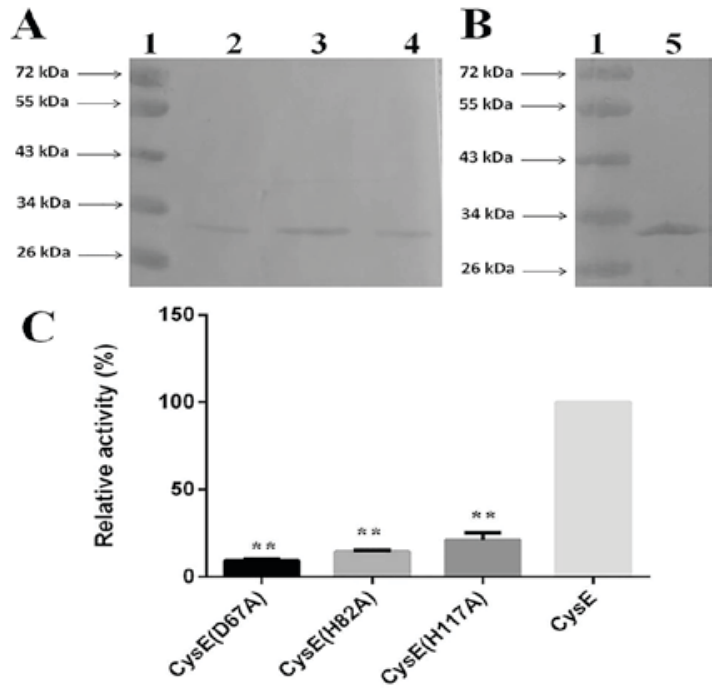

Figure 3. The identification of purified mutated CysE protein and serine acetyltransferase activity (A and B) Western blot analysis of purified mutated CysE proteins from E. coli BL21 (DE3). Lane 1, protein marker; lane 2, mutated D67A CysE protein; lane 3, mutated H82A CysE; lane 4, mutated H117A CysE protein ; lane 5, wild-type CysE protein. (C) Relative enzymatic activity of CysE with different mutated sites compared with wild-type CysE $\left({ }^{* *} \mathrm{P}<0.01\right)$. CysE, serine acetyltransferase.

structure and function. The SWISS-MODEL server was used to model proteins based on protein-structure homology (24). A PSI-BLAST search revealed 3P47A as an available template that can be used for homology modeling of the CysE protein. 3P47A is a refined X-ray diffraction model of the A-chain of E. histolytica CysE at a resolution of $1.78 \AA$. Homology modeling of $M$. tuberculosis CysE was constructed using 3P47A as a template (Fig. 2A).

Energy minimization, quality assessment and visualization. The reliability of the predicted 3D model of the M. tuberculosis CysE was assessed by Ramachandran plot, using PROCHECK, and a quality assessment confirmed using PDBsum. The Ramachandran plot revealed a distribution of 160 amino acid residues of M. tuberculosis CysE, with the majority of residues $(98.4 \%)$ in the favored and allowed regions, and only two of the residues $(1.6 \%)$ in the generously allowed region (Fig. 2B). The $g$-factor demonstrates how unusual a stereochemical property is: Values below -0.5 represent unusual property, whereas values below -1.0 indicate high unusualness. The $g$-factors for dihedral angles and main chain covalent forces were -0.10 and 0.31 , respectively. The overall average $g$-factor for the M. tuberculosis CysE model was 0.07. Taken together, the Ramachandran plot and $g$-factors indicated that the backbone dihedral angles, phi and psi in the 3D model of M. tuberculosis CysE were within acceptable limits.

Following the construction of a predicted 3D structure, the final protein model was verified using Verify3D, which analyzed the compatibility of the 3D (atomic) model of CysE with its own amino acid sequence (1D). The scores of a sliding 21-residue window (between -10 and +10 ) were added and plotted for the individual residues $(25,26)$. The M.tuberculosis CysE protein was consistent with the respective amino acids, with $88.82 \%$ of the residues having an averaged 3D-1D and a 
score $>0.2$. ERRAT is a protein structure verification algorithm that is well suited for evaluating the quality of crystallographic model building and refinement. The final model had a quality factor of $91.156 \%$ in ERRAT, indicating that the model was acceptable and credible.

Site-directed mutagenesis of M. tuberculosis CysE. The conserved residues D67, H82 and $\mathrm{H} 117$ of M. tuberculosis CysE were selected for site-directed mutation. To maintain the native spatial structure, these three residues were substituted with alanine. Three linear pMD18 plasmid vectors containing the mutated $M$. tuberculosis cysE gene (pMD18-Mtb-cysE) were amplified using mutated primers. Restriction endonuclease digestion and sequencing analysis confirmed that the three specific site-directed mutations were obtained successfully (data not shown).

Preparation of mutant M. tuberculosis CysE proteins. The pET29b-Mtb-cysE expression plasmids containing the subcloned site-directed mutations were constructed and transformed into E. coli. Three different soluble CysE mutant proteins with fused His-tags were expressed in E. coli BL21 (DE3) cells, and purified by $\mathrm{Ni}^{2+}$ affinity chromatography. The purified mutant proteins were identified by western blot analysis. The band of CysE mutant proteins appeared at $\sim 30 \mathrm{kDa}$, which was similar to that of wild-type M. tuberculosis CysE protein (7) (Fig. 3A and B).

Enzymatic activity assay for mutant CysE proteins. CysE enzymatic activity of the three mutant proteins was detected using a colorimetric assay with DTNB (19), which indicated that the mutant $M$. tuberculosis CysE proteins exhibited different enzymatic activity compared with wild-type CysE. The D67A mutant demonstrated only $9.79 \%$ activity, whereas the H82A and H117A mutants had 14.31 and $20.82 \%$ activity, respectively (Fig. 3C). These data suggested that the three residues may be involved in the functional activity of the enzyme.

In conclusion, site-directed mutagenesis of CysE and the enzymatic activity assay indicated that the conserved amino acid residues D67, H82 and H117 may be involved in the catalytic activity and, thus, the function of CysE. The prediction for M. tuberculosis CysE structure may facilitate the elucidation of the functional mechanism of this enzyme. However, further studies regarding the interaction between Cys as a substrate and M. tuberculosis CysE as an enzyme are required. Furthermore, determination of the active amino acids of M. tuberculosis CysE will immensely promote the development of structure-guided enzyme inhibitors for use as novel and effective anti-TB drugs.

\section{Acknowledgements}

The present study was supported by the National Natural Science Foundation of China (grant no. 31070066).

\section{References}

1. Cole ST and Riccardi G: New tuberculosis drugs on the horizon. Curr Opin Microbiol 14: 570-576, 2011.
2. Hell R: Molecular physiology of plant sulfur metabolism. Planta 202: 138-148, 1997.

3. Kredich NM, Becker MA and Tomkins GM: Purification and characterization of cysteine synthetase, a bifunctional protein complex, from Salmonella typhimurium. J Biol Chem 244: 2428-2439, 1969.

4. Kredich NM and Tomkins GM: The enzymic synthesis of L-cysteine in Escherichia coli and Salmonella typhimurium. J Biol Chem 241: 4955-4965, 1966.

5. Schnell R and Schneider G: Structural enzymology of sulphur metabolism in Mycobacterium tuberculosis. Biochem Biophys Res Commun 396: 33-38, 2010.

6. Raman K, Yeturu K and Chandra N: targetTB: A target identification pipeline for Mycobacterium tuberculosis through an interactome, reactome and genome-scale structural analysis. BMC Syst Biol 2: 109, 2008.

7. Qiu J, Wang D, Ma Y, Jiang T and Xin Y: Identification and characterization of serine acetyltransferase encoded by the Mycobacterium tuberculosis Rv2335 gene. Int J Mol Med 31: 1229-1233, 2013.

8. Benson DA, Karsch-Mizrachi I, Lipman DJ, Ostell J and Wheeler DL: GenBank. Nucleic Acids Res 36 (Database Issue): D25-D30, 2008.

9. Wilkins MR, Gasteiger E, Bairoch A, Sanchez JC, Williams KL, Appel RD and Hochstrasser DF: Protein identification and analysis tools in the ExPASy server. Methods Mol Biol 112: 531-552, 1999.

10. Corpet F: Multiple sequence alignment with hierarchical clustering. Nucleic Acids Res 16: 10881-10890, 1988.

11. Zdobnov EM and Apweiler R: InterProScan-an integration platform for the signature-recognition methods in InterPro. Bioinformatics 17: 847-848, 2001.

12. McGuffin LJ, Bryson K and Jones DT: The PSIPRED protein structure prediction server. Bioinformatics 16: 404-405, 2000.

13. Marchler-Bauer A, Derbyshire MK, Gonzales NR, Lu S Chitsaz F, Geer LY, Geer RC, He J, Gwadz M, Hurwitz DI, et al: CDD: NCBI's conserved domain database. Nucleic Acids Res 43 (Database Issue): D222-D226, 2015.

14. Guex N, Peitsch MC and Schwede T: Automated comparative protein structure modeling with SWISS-MODEL and Swiss-PdbViewer: A historical perspective. Electrophoresis 30 (Suppl 1): S162-S173, 2009.

15. Altschul SF, Madden TL, Schäffer AA, Zhang J, Zhang Z, Miller W and Lipman DJ: Gapped BLAST and PSI-BLAST: A new generation of protein database search programs. Nucleic Acids Res 25: 3389-3402, 1997.

16. Guex N and Peitsch MC: SWISS-MODEL and the Swiss-PdbViewer: An environment for comparative protein modeling. Electrophoresis 18: 2714-2723, 1997.

17. Laskowski RA: PDBsum: Summaries and analyses of PDB structures. Nucleic acids Res 29: 221-222, 2001.

18. Laskowski RA, Rullmannn JA, MacArthur MW, Kaptein R and Thornton JM: AQUA and PROCHECK-NMR: Programs for checking the quality of protein structures solved by NMR. J Biomol NMR 8: 477-486, 1996.

19. Riddles PW, Blakeley RL and Zerner B: Reassessment of Ellman's reagent. Methods Enzymol 91: 49-60, 1983.

20. Downie JA: The nodL gene from Rhizobium leguminosarum is homologous to the acetyl transferases encoded by lacA and cysE. Mol Microbiol 3: 1649-1651, 1989.

21. Vaara M: Eight bacterial proteins, including UDP-N-acetylglucosamine acyltransferase (LpxA) and three other transferases of Escherichia coli, consist of a six-residue periodicity theme. FEMS Microbiol Lett 76: 249-254, 1992.

22. Jenkins $J$ and Pickersgill R: The architecture of parallel beta-helices and related folds. Prog Biophys Mol Biol 77: 111-175, 2001.

23. Raetz CR and Roderick SL: A left-handed parallel beta helix in the structure of UDP-N-acetylglucosamine acyltransferase. Science 270: 997-1000, 1995 .

24. Arnold K, Bordoli L, Kopp J and Schwede T: The SWISS-MODEL workspace: A web-based environment for protein structure homology modelling. Bioinformatics 22: 195-201, 2006.

25. Luthy R, Bowie JU and Eisenberg D: Assessment of protein models with three-dimensional profiles. Nature 356: 83-85, 1992.

26. Bowie JU, Luthy R and Eisenberg D: A method to identify protein sequences that fold into a known three-dimensional structure. Science 253: 164-170, 1991. 